\title{
SPATIAL SYNCHRONIZATION OF UNBALANCED ROTORS EXCITED WITH PARALLELED AND COUNTERROTATING MOTORS IN A FAR RESONANCE SYSTEM
}

\author{
PAN FANG \\ School of Mechanical Engineering, Southwest Petroleum University, Chengdu, China \\ Key Laboratory of Oil $\&$ Gas Equipment, Ministry of Education, Southwest Petroleum University, Chengdu, China \\ School of Petroleum Engineering, Southwest Petroleum University, Chengdu, China \\ e-mail: ckfangpan@126.com \\ Min Zou, Huan Peng, Minguun Du, Gang Hu, Yonguun Hou \\ School of Mechanical Engineering, Southwest Petroleum University, Chengdu, China
}

\begin{abstract}
Dynamic characteristics of the vibration screening machinery is influenced by synchronization between induction motors. Therefore, estimating the synchronous state between the motors is a crucial process for designing the vibration screening machinery. In this paper, two rotors excited with paralleled and counterrotating motors in a far resonance system are concerned. To master the synchronization of the system, the dynamic model is firstly established; then, the synchronous condition of the system is derived with the Poincaré method; subsequently, the synchronous stability of the system is discussed by the Hamilton principle; finally, some computation simulations are implemented to verify correctness of theoretical analysis. The research result shows that the system actuated by rotors of the identical mass is planar motion as the stable phase difference between the rotors is stabilized in the zero phase. The system actuated by nonequivalent mass rotors exhibits spatial motion as the stable phase difference stabilizes in a nonzero phase.
\end{abstract}

Keywords: synchronization, rotors, dynamics, stability

\section{Introduction}

The synchronization phenomenon, defined by Huygens in 1665 through of observation motion of two pendulum clocks hung in an elastic beam, is widely appeared in the world, such as music synchronization in concert, communication synchronization in networks and flight synchronization of wild geese (Banaszewski and Schollbach, 1998; Xiao et al., 2017; Tang et al., 2019). Blekhman (1988) found synchronization of a dual-motor excitation in a non-resonant system, and proposed synchronization theory of the dual-motor system with the Poincaré-Lyapunov method, which guides the application of synchronization theory in theoretical research and practical engineering. Czolczynski et al. (2012, 2013) and Kapitaniak et al. (2014) considered synchronization of a series of pendula installed on a horizontal beam, and the synchronous condition of the system was derived by analytical methods and numerical computations. Those researches revealed synchronous characteristics in nonlinear dynamic systems. Inoue et al. (1951) gave a detailed description for synchronization of the dual-rotor excitation in a frequency-tripled vibration system. Wen et al. (2009) used the Hamilton theory and average method to deduce conditions of synchronization and synchronous stability. The core idea of these methods is seeking the balanced torque equation between the motor shafts in the synchronous state. The author also described that the frequency-triple and frequency-multiple synchronization exists in 
a certain vibrating system. Then, Zhao et al. (2010a,b) studied a revised small parameter method to describe the synchronous process of rotor systems, which greatly simplifies the solving process of the synchronization problem in rotor systems. Based on Zhao's method, Zhang et al. $(2014,2016,2017)$ discussed the transmission mechanism of synchronous torques between a cylindrical roller and a multi-rotor in a vibrating system. The author showed that the greater value of the maximum of the synchronous torques, the greater the allowed difference of residual torque between motors accomplishing synchronous operation of unbalanced rotors. Besides, Balthazar et al. $(2004,2005)$ proposed some short comments on synchronous rotation of two (or four) non-ideal unbalanced rotors supported by a flexible strut structure. In a particular case the "Sommerfeld effect" was also considered. Nanha Djanan et al. (2013, 2014) explored the system in which three motors worked on the same plate, and the synchronous operation of the rotors depended on physical characteristics of the motors and the plate. Fang et al. (2014), Hou et al. (2018), Fang and Hou (2018) considered synchronization and stability of an elastically coupled rotors in vibration systems, and found that synchronous characteristics of the system were also influenced by stiffness of coupling springs. Sperling et al. (2000) presented a two-plane automatic balancing device for equilibration of rigid-rotor unbalance. They did not only limit to report results of numerical simulation, but also contained derivation of equation of motion for the considered system as well as analysed of stability conditions on the basis of an analytical approximation. In light of an active control strategy, Kong et al. (2016a,b) implemented the synchronous state with the zero phase between three rotors by employing an adaptive sliding mode algorithm to control three motors. The studies above were mainly related to synchronization of mechanical systems in a two-dimensional surface, that is to say, the objects of vibratory synchronization transmission vibrated in a plane. Paz and Cole (1992) and Zhao et al. (2010a,b) discussed spatial synchronization of a vibrating spiral elevator excited by two perpendicular unbalanced rotors as spatial motion of the elevator. Chen et al. (2016) gave theoretical and experimental descriptions to spatial synchronization of two eccentric rotors with a common rotational axis. These research studies promoted development of separating technology of the screening machinery and settlement of the common scientific issue of vibrating synchronization.

In this paper, the dynamic model stems from the linear screening machine, which is used to screen solid particles from the drilling fluid in drilling engineering. Dynamic characteristics of the vibration screening machine are determined by synchronization of motors. An interesting phenomenon is that the vibration locus of this screening machine is linear in a plane when the rotors of identical mass operate in the opposite direction, which is in favor of transport of solid particles in the drilling fluid. However, when unbalanced rotors of different mass operate in the opposite direction, it becomes intractable to convey solid particles. In this paper, we will explain why this phenomenon happens.

\section{Dynamic model}

From Fig. 1, it follows that the vibration screening machine consists of an induction motor, vibrating body, elastic element and foundation support, as shown in Fig. 1. A rigid vibrating body of mass $m_{3}$ is elastically supported via linear damping springs with stiffness $k_{j}$ and damping $f_{j}$ in the $j$-direction $(j=x, y, z, \psi, \delta, v)$. The unbalanced rotor $i$ actuated by the induction motor is modelled by a point mass $m_{i}$ (for $i=1,2$ ) with eccentricity radius $r$.

The transformation of reference coordinates is shown in Fig. 1b, and conversion sequence of the reference coordinates is followed by $\left(G x^{\prime \prime \prime} y^{\prime \prime \prime} z^{\prime \prime \prime}\right) \rightarrow\left(G x^{\prime \prime} y^{\prime \prime} z^{\prime \prime}\right) \rightarrow\left(G x^{\prime} y^{\prime} z^{\prime}\right)$. The cosine matrices of rotation direction corresponding to the coordinates can be expressed by 

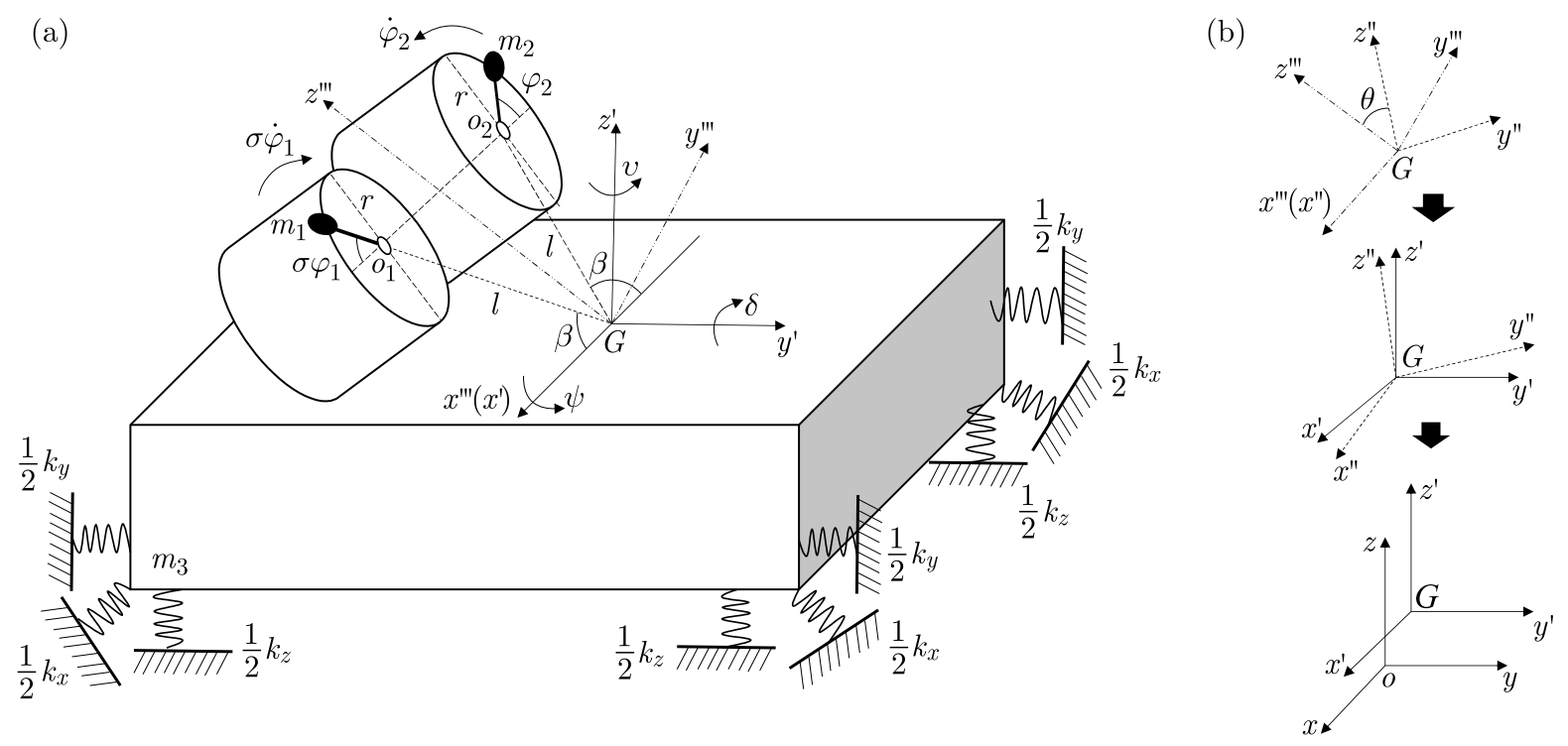

Fig. 1. Dynamic model of the vibration screening machinery; (a) dynamic model, (b) reference coordinates

$$
\begin{array}{rlrl}
\mathbf{A}_{1}= & {\left[\begin{array}{ccc}
1 & 0 & 0 \\
0 & -\cos \theta & \sin \theta \\
0 & \sin \theta & -\cos \theta
\end{array}\right]} & \mathbf{A}_{2}=\left[\begin{array}{ccc}
1 & 0 & 0 \\
0 & \cos \psi & -\sin \psi \\
0 & \sin \psi & \cos \psi
\end{array}\right] \\
\mathbf{A}_{3}=\left[\begin{array}{ccc}
\cos \delta & 0 & \sin \delta \\
0 & 1 & 0 \\
-\sin \delta & 0 & \cos \delta
\end{array}\right] & \mathbf{A}_{4}=\left[\begin{array}{ccc}
\cos v & -\sin v & 0 \\
\sin v & \cos v & 0 \\
0 & 0 & 1
\end{array}\right]
\end{array}
$$

Considering infinitesimal rotation of the vibrating body in $\psi$-, $\delta$ - and $v$-directions, matrices $\mathbf{A}_{2}, \mathbf{A}_{3}$ and $\mathbf{A}_{4}$ are simplified as

$$
\mathbf{A}_{2}=\left[\begin{array}{ccc}
1 & 0 & 0 \\
0 & 1 & -\psi \\
0 & \psi & 1
\end{array}\right] \quad \mathbf{A}_{3}=\left[\begin{array}{ccc}
1 & 0 & \delta \\
0 & 1 & 0 \\
-\delta & 0 & 1
\end{array}\right] \quad \mathbf{A}_{4}=\left[\begin{array}{ccc}
1 & -v & 0 \\
v & 1 & 0 \\
0 & 0 & 1
\end{array}\right]
$$

In the rotating coordinate system $G x^{\prime \prime \prime} y^{\prime \prime \prime} z^{\prime \prime \prime}$, the centroid coordinates of unbalanced rotors 1 and 2 is written by

$$
\mathbf{x}_{1}^{\prime \prime \prime}=\left[\begin{array}{c}
l \cos \beta+r \cos \varphi_{1} \\
0 \\
l \sin \beta+r \sin \varphi_{1}
\end{array}\right] \quad \mathbf{x}_{2}^{\prime \prime \prime}=\left[\begin{array}{c}
-l \cos \beta+r \cos \varphi_{2} \\
0 \\
l \sin \beta+r \sin \varphi_{2}
\end{array}\right]
$$

The centroid coordinates of the unbalanced rotors in $G x^{\prime} y^{\prime}$ can be obtained through transformation of the rotation matrix $\mathbf{R}$. Centroid displacements of the vibrating body in $O x y z$ is assumed as $\mathbf{x}_{G}=[x, y, z]^{\mathrm{T}}$, and so the centroid of the rotors in the coordinates $O x y$ can be expressed by

$$
\begin{aligned}
& \mathbf{x}_{1}=\mathbf{x}_{G}+\mathbf{A}_{1} \mathbf{R} \mathbf{x}_{1}^{\prime \prime \prime} \\
& \mathbf{x}_{2}=\mathbf{x}_{G}+\mathbf{A}_{1} \mathbf{R}_{2}^{\prime \prime \prime}
\end{aligned}
$$

where

$$
\mathbf{R}=\mathbf{A}_{2} \mathbf{A}_{3} \mathbf{A}_{4}=\left[\begin{array}{ccc}
1 & -v & \delta \\
v & 1 & -\psi \\
-\delta & \psi & 1
\end{array}\right]
$$


According to kinetic theory, kinetic energy of the system $T$ can be written as in the following

$$
T=\frac{1}{2} m_{3}\left(\dot{x}^{2}+\dot{y}^{2}+\dot{z}^{2}\right)+\frac{1}{2} J_{\psi} \dot{\psi}^{2}+\frac{1}{2} J_{\delta} \dot{\delta}^{2}+\frac{1}{2} J_{v} \dot{v}^{2}+\frac{1}{2} \sum_{i=1}^{2} J_{i} \dot{\varphi}_{i}^{2}+\frac{1}{2} \sum_{i=1}^{2} m_{i} \dot{\mathbf{x}}_{i}^{\mathrm{T}} \dot{\mathbf{x}}_{i}
$$

In addition, potential energy of the system $V$ can be expressed by

$$
V=\frac{1}{2} k_{x} x^{2}+\frac{1}{2} k_{y} y^{2}+\frac{1}{2} k_{z} z^{2}+\frac{1}{2} k_{\psi} \psi^{2}+\frac{1}{2} k_{\delta} \delta^{2}+\frac{1}{2} k_{v} v^{2}
$$

Moreover, dissipated energy of the system $D$ can be obtained by

$$
D=\frac{1}{2} f_{x} \dot{x}^{2}+\frac{1}{2} f_{y} \dot{y}^{2}+\frac{1}{2} f_{z} \dot{z}^{2}+\frac{1}{2} f_{\psi} \dot{\psi}^{2}+\frac{1}{2} f_{\delta} \dot{\delta}^{2}+\frac{1}{2} f_{v} \dot{v}^{2}
$$

According to the Lagrange equation

$$
\frac{d}{d t}\left(\frac{\partial T}{\partial \dot{q}}\right)-\frac{\partial(T-V)}{\partial q}+\frac{\partial D}{\partial \dot{q}}=Q
$$

differential equations of motion of the system can be derived. In the system, a matrix $\mathbf{q}=\left[x, y, z, \psi, \delta, v, \varphi_{1}, \varphi_{2}\right]^{\mathrm{T}}$ is chosen as the generalized coordinate matrix, and the generalized force matrix of the vibrating system is given by

$$
\left[\begin{array}{llllllll}
Q_{x} & Q_{y} & Q_{z} & Q_{\psi} & Q_{\delta} & Q_{v} & Q_{\varphi_{1}} & Q_{\varphi_{2}}
\end{array}\right]^{\mathrm{T}}=\left[\begin{array}{llllllll}
0 & 0 & 0 & 0 & 0 & 0 & M_{e 1}-R_{e 1} & M_{e 2}-R_{e 2}
\end{array}\right]^{\mathrm{T}}
$$

Substituting Eqs. (2.5), (2.6), (2.7) and (2.9) into Eq. (2.8), the dynamic equation of the system is written by

$$
\begin{aligned}
& M \ddot{x}+f_{x} \dot{x}+k_{x} x=m_{1} r\left(\ddot{\varphi}_{1} \sin \varphi_{1}+\dot{\varphi}_{1}^{2} \cos \varphi_{1}\right)-m_{2} r\left(\ddot{\varphi}_{2} \sin \varphi_{2}+\dot{\varphi}_{2}^{2} \cos \varphi_{2}\right) \\
& M \ddot{y}+f_{y} \dot{y}+k_{y} y=m_{1} r \sin \theta\left(\dot{\varphi}_{1}^{2} \sin \varphi_{1}-\ddot{\varphi}_{1} \cos \varphi_{1}\right)+m_{2} r \sin \theta\left(\dot{\varphi}_{2}^{2} \sin \varphi_{2}-\ddot{\varphi}_{2} \cos \varphi_{2}\right) \\
& M \ddot{z}+f_{z} \dot{z}+k_{z} z=m_{1} r \cos \theta\left(\ddot{\varphi}_{1} \cos \varphi_{1}-\dot{\varphi}_{1}^{2} \sin \varphi_{1}\right)+m_{2} r \cos \theta\left(\ddot{\varphi}_{2} \cos \varphi_{2}-\dot{\varphi}_{2}^{2} \sin \varphi_{2}\right) \\
& J_{\psi} \ddot{\psi}+f_{\psi} \dot{\psi}+k_{\psi} \psi=m_{1} r l \sin 2 \theta \sin \beta\left(\dot{\varphi}_{1}^{2} \sin \varphi_{1}-\ddot{\varphi}_{1} \cos \varphi_{1}\right) \\
& \quad+m_{2} r l \sin 2 \theta \sin \beta\left(\dot{\varphi}_{2}^{2} \sin \varphi_{2}-\ddot{\varphi}_{2} \cos \varphi_{2}\right) \\
& J_{\delta} \ddot{\delta}+f_{\delta} \dot{\delta}+k_{\delta} \delta=m_{1} r l\left[\ddot{\varphi}_{1} \cos \left(\varphi_{1}-\beta\right)-\dot{\varphi}_{1}^{2} \sin \left(\varphi_{1}-\beta\right)\right] \\
& \quad-m_{2} r l\left[\ddot{\varphi}_{2} \cos \left(\varphi_{2}-\beta\right)-\dot{\varphi}_{2}^{2} \sin \left(\varphi_{2}-\beta\right)\right] \\
& J_{v} \ddot{v}+f_{v} \dot{v}+k_{v} v=m_{1} r l \sin 2 \theta \cos \beta\left(\ddot{\varphi}_{1} \cos \varphi_{1}-\dot{\varphi}_{1}^{2} \sin \varphi_{1}\right) \\
& \quad-m_{2} r l \sin 2 \theta \cos \beta\left(\ddot{\varphi}_{2} \cos \varphi_{2}-\dot{\varphi}_{2}^{2} \sin \varphi_{2}\right) \\
& J_{1} \ddot{\varphi}_{1}=M_{e 1}-R_{e 1}+m_{1} r \sin \varphi_{1}(\ddot{x}+l \ddot{\delta} \sin \beta) \\
& \quad+m_{1} r \cos \varphi_{1}(\ddot{z} \cos \theta-\ddot{y} \sin \theta+l \ddot{v} \cos \beta \sin 2 \theta+l \ddot{\delta} \cos \beta-l \ddot{\psi} \sin \beta \sin 2 \theta) \\
& J_{2} \ddot{\varphi}_{2}=M_{e 2}-R_{e 2}-m_{2} r \sin \varphi_{2}(\ddot{x}+l \ddot{\delta} \sin \beta) \\
& \quad+m_{2} r \cos \varphi_{2}(\ddot{z} \cos \theta-\ddot{y} \sin \theta-l \ddot{v} \cos \beta \sin 2 \theta-l \ddot{\delta} \cos \beta-l \ddot{\psi} \sin \beta \sin 2 \theta)
\end{aligned}
$$

In the synchronous or steady state, velocity of the motors is stabilized at its rated speed, hence, acceleration of the motors $\ddot{\varphi}_{i}$ is approximately equal to zero. Therefore, introducing a small parameter $\mu$, the Poincaré form of the last two formulas of Eq. (2.10) can be given (Fang et al., 2014; Fang and Hou, 2018; Hou et al., 2018)

$$
J_{1} \ddot{\varphi}_{1}=\mu \Phi_{1} \quad J_{2} \ddot{\varphi}_{2}=\mu \Phi_{2}
$$


where

$$
\begin{aligned}
& \mu \Phi_{1}=M_{e 1}-R_{e 1}+m_{1} r \sin \varphi_{1}(\ddot{x}+\ddot{\delta} l \sin \beta) \\
& \quad+m_{1} r \cos \varphi_{1}(\ddot{z} \cos \theta-\ddot{y} \sin \theta+l \ddot{v} \cos \beta \sin 2 \theta+l \ddot{\delta} \cos \beta-l \ddot{\psi} \sin \beta \sin 2 \theta) \\
& \mu \Phi_{2}=M_{e 2}-R_{e 2}-m_{2} r \sin \varphi_{2}(\ddot{x}+\ddot{\delta} l \sin \beta) \\
& \quad+m_{2} r \cos \varphi_{2}(\ddot{z} \cos \theta-\ddot{y} \sin \theta-l \ddot{v} \cos \beta \sin 2 \theta-l \ddot{\delta} \cos \beta-l \ddot{\psi} \sin \beta \sin 2 \theta)
\end{aligned}
$$

\section{Synchronism of two rotors}

In th steady state of the vibrating system, accelerations of the motors $\ddot{\varphi}_{1}$ and $\ddot{\varphi}_{2}$ are approximately equal to zero, and velocities of the motors $\dot{\varphi}_{1}$ and $\dot{\varphi}_{2}$ are stabilized at the rated speed $\omega$, i.e., $\ddot{\varphi}_{1}=\ddot{\varphi}_{2}=0$ and $\dot{\varphi}_{1}=\dot{\varphi}_{2}=\omega$. In order to obtain the non-dimensional approximate solution of the system, the following dimensionless parameters

$$
\begin{array}{lcrl}
\eta_{1}=\frac{m_{1}}{M} & \eta_{2}=\frac{m_{2}}{M} & \\
\zeta_{x}=\frac{f_{x}}{2 \sqrt{M k_{x}}} & \zeta_{y}=\frac{f_{y}}{2 \sqrt{M k_{y}}} & \zeta_{z}=\frac{f_{z}}{2 \sqrt{M k_{z}}} & \\
\zeta_{\psi}=\frac{f_{\psi}}{2 \sqrt{J_{\psi} k_{\psi}}} & \zeta_{\delta}=\frac{f_{\delta}}{2 \sqrt{J_{\delta} k_{\delta}}} & \zeta_{v}=\frac{f_{v}}{2 \sqrt{J_{v} k_{v}}} & r_{l}=\frac{l}{l_{e}}
\end{array}
$$

are introduced into Eq. (2.10), and so the first six formulas in Eq. (2.10) can be rewritten as

$$
\begin{aligned}
& \ddot{x}+2 \zeta_{x} \omega_{x} \dot{x}+\omega_{x}^{2} x=\omega^{2}\left(\eta_{1} r \cos \varphi_{1}-\eta_{2} r \cos \varphi_{2}\right) \\
& \ddot{y}+2 \zeta_{y} \omega_{y} \dot{y}+\omega_{y}^{2} y=\omega^{2}\left(\eta_{1} r \sin \theta \sin \varphi_{1}+\eta_{2} r \sin \theta \sin \varphi_{2}\right) \\
& \ddot{z}+2 \zeta_{z} \omega_{z} \dot{z}+\omega_{z}^{2} z=\omega^{2}\left(\eta_{1} r \cos \theta \sin \varphi_{1}+\eta_{2} r \cos \theta \sin \varphi_{2}\right) \\
& \ddot{\psi}+2 \zeta_{\psi} \omega_{\psi} \dot{\psi}+\omega_{\psi}^{2} \psi=\omega^{2}\left(\frac{\eta_{1} r r_{l} \sin 2 \theta \sin \beta}{l_{e}} \sin \varphi_{1}+\frac{\eta_{2} r r_{l} \sin 2 \theta \sin \beta}{l_{e}} \sin \varphi_{2}\right) \\
& \ddot{\delta}+2 \zeta_{\delta} \omega_{\delta} \dot{\delta}+\omega_{\delta}^{2} \delta=\omega^{2}\left[\frac{-\eta_{1} r r_{l}}{l_{e}} \sin \left(\varphi_{1}-\beta\right)+\frac{\eta_{2} r r_{l}}{l_{e}} \sin \left(\varphi_{2}-\beta\right)\right] \\
& \ddot{v}+2 \zeta_{v} \omega_{v} \dot{v}+\omega_{v}^{2} v=\omega^{2}\left[\frac{-\eta_{1} r r_{l} \sin 2 \theta \cos \beta}{l_{e}} \sin \varphi_{1}+\frac{\eta_{2} r r_{l} \sin 2 \theta \cos \beta}{l_{e}} \sin \varphi_{2}\right]
\end{aligned}
$$

where

$$
\begin{array}{ll}
\omega_{x}=\sqrt{\frac{k_{x}}{M}} \quad \omega_{y}=\sqrt{\frac{k_{y}}{M}} \quad \omega_{z}=\sqrt{\frac{k_{z}}{M}} \omega_{\psi}=\sqrt{\frac{k_{\psi}}{J_{\psi}}} \quad \omega_{\delta}=\sqrt{\frac{k_{\delta}}{J_{\delta}}} \\
\omega_{v}=\sqrt{\frac{k_{v}}{J_{v}}} \quad l_{e}=\sqrt{\frac{J_{\psi}}{M}} \approx \sqrt{\frac{J_{\delta}}{M}} \approx \sqrt{\frac{J_{v}}{M}}
\end{array}
$$

Thus, the displacement responses of the vibrating body can be expressed as follows

$$
\begin{aligned}
x & =r \mu_{x}\left[\eta_{1} \cos \left(\varphi_{1}-\gamma_{x}\right)-\eta_{2} \cos \left(\varphi_{2}-\gamma_{x}\right)\right] \\
y & =r \mu_{y} \sin \theta\left[\eta_{1} \sin \left(\varphi_{1}-\gamma_{y}\right)+\eta_{2} \sin \left(\varphi_{2}-\gamma_{y}\right)\right] \\
z & =r \mu_{z} \cos \theta\left[\eta_{1} \sin \left(\varphi_{1}-\gamma_{z}\right)+\eta_{2} \sin \left(\varphi_{2}-\gamma_{z}\right)\right] \\
\psi & =\frac{r r_{l} \mu_{\psi} \sin 2 \theta \sin \beta}{l_{e}}\left[\eta_{1} \sin \left(\varphi_{1}-\gamma_{\psi}\right)+\eta_{2} \sin \left(\varphi_{2}-\gamma_{\psi}\right)\right] \\
\delta & =\frac{r r_{l} \mu_{\delta}}{l_{e}}\left[\eta_{2} \sin \left(\varphi_{2}-\gamma_{\delta}-\beta\right)-\eta_{1} \sin \left(\varphi_{1}-\gamma_{\delta}-\sigma \beta\right)\right] \\
v & =\frac{r r_{l} \mu_{v} \sin 2 \theta \cos \beta}{l_{e}}\left[\eta_{2} \sin \left(\varphi_{2}-\gamma_{v}\right)-\eta_{1} \sin \left(\varphi_{1}-\gamma_{v}\right)\right]
\end{aligned}
$$


where $\mu_{j}=1 / \sqrt{\left(1-n_{j}^{2}\right)^{2}+\left(2 \zeta_{j} n_{j}\right)^{2}},(j=x, y, z, \psi, \delta, v)$, and $\gamma_{j}=\tan ^{-1}\left(2 \zeta_{j} n_{j}\right) /\left(1-n_{j}^{2}\right), \gamma_{j}$ are lagging phase angles in the $j$-direction, in which $n_{j}=\omega_{j} / \omega$.

\subsection{Synchronous condition}

Based on the approximate solutions above, the synchronous condition and synchronous stability between the rotors can be determined with the Poincaré method (Fang and Hou, 2018). In addition, the phase angle of the rotors can be assumed as

$$
\varphi_{1}=\omega t+\alpha_{1} \quad \varphi_{2}=\omega t+\alpha_{2}
$$

When the asynchronous motors operate in the steady state, the phase difference between the rotors is noted as

$$
\alpha=\alpha_{1}-\alpha_{2}
$$

According to Eq. (3.3), the two-order derivative of $x, y, z, v, \delta$ and $\psi$ with respect to time $t$ can be calculated. Substituting $\ddot{x}, \ddot{y}, \ddot{z}, \ddot{v}, \ddot{\delta}$ and $\ddot{\psi}$ into Eq. (2.12), and then integrating and averaging this equation related to $t$ over period $T$, respectively, synchronous indexes $P_{1}$ and $P_{2}$ can be obtained

$$
\begin{aligned}
& P_{1}=\frac{1}{T} \int_{0}^{T} \mu \Phi_{1} d t=\bar{M}_{e 1}-\bar{R}_{e 1}+\frac{1}{2} m r^{2} \omega^{2}\left[\eta_{1}^{2} W_{s 0}+W_{s} \cos \bar{\alpha}+W_{c} \sin \bar{\alpha}\right] \\
& P_{2}=\frac{1}{T} \int_{0}^{T} \mu \Phi_{2} d t=\bar{M}_{e 2}-\bar{R}_{e 2}+\frac{1}{2} M r^{2} \omega^{2}\left[\eta_{2}^{2} W_{s 0}+W_{s} \cos \bar{\alpha}-W_{c} \sin \bar{\alpha}\right]
\end{aligned}
$$

where

$$
\begin{aligned}
W_{s 0} & =-\mu_{x} \sin \gamma_{x}-\mu_{y} \sin ^{2} \theta \sin \gamma_{y}+\mu_{z} \cos ^{2} \theta \sin \gamma_{z} \\
& -\mu_{\psi} r_{l}^{2} \sin ^{2} 2 \theta \sin ^{2} \beta \sin \gamma_{\psi}-\mu_{v} r_{l}^{2} \sin ^{2} 2 \theta \cos ^{2} \beta \sin \gamma_{v}-\mu_{\delta} r_{l}^{2} \sin \gamma_{\delta} \\
W_{s} & =\eta_{1} \eta_{2} \mu_{x} \sin \gamma_{x}-\eta_{1} \eta_{2} \mu_{y} \sin ^{2} \theta \sin \gamma_{y}+\eta_{1} \eta_{2} \mu_{z} \cos ^{2} \theta \sin \gamma_{z} \\
& -\eta_{1} \eta_{2} \mu_{\psi} r_{l}^{2} \sin ^{2} 2 \theta \sin ^{2} \beta \sin \gamma_{\psi}+\eta_{1} \eta_{2} \mu_{v} r_{l}^{2} \sin ^{2} 2 \theta \cos ^{2} \beta \sin \gamma_{v}+\eta_{1} \eta_{2} \mu_{\delta} r_{l}^{2} \sin \gamma_{\delta} \\
W_{c} & =\eta_{1} \eta_{2} \mu_{x} \cos \gamma_{x}-\eta_{1} \eta_{2} \mu_{y} \sin ^{2} \theta \cos \gamma_{y}+\eta_{1} \eta_{2} \mu_{z} \cos ^{2} \theta \cos \gamma_{z} \\
& -\eta_{1} \eta_{2} \mu_{\psi} r_{l}^{2} \sin ^{2} 2 \theta \sin ^{2} \beta \cos \gamma_{\psi}+\eta_{1} \eta_{2} \mu_{v} r_{l}^{2} \sin ^{2} 2 \theta \cos ^{2} \beta \cos \gamma_{v}+\eta_{1} \eta_{2} \mu_{\delta} r_{l}^{2} \cos \gamma_{\delta}
\end{aligned}
$$

As shown in Eq. (3.7), the values $W_{s 0}$ and $W_{s}$ are defined as sine coefficients of the lagging phase $\gamma_{j}(j=x, y, z, \psi, \delta, v)$ and $W_{c}$ are denoted as cosine coefficients of the lagging phase $\gamma_{j}$.

Now, the possible synchronous motion of the system is analyzed. We find from Eqs. (3.6) and (3.7)

$$
\begin{aligned}
& \left(\bar{M}_{e 1}+\bar{M}_{e 2}\right)-\left(\bar{R}_{e 1}+\bar{R}_{e 2}\right)+\frac{1}{2} M r^{2} \omega^{2}\left[\left(\eta_{1}^{2}+\eta_{2}^{2}\right) W_{s 0}+2 W_{s} \cos \alpha\right]=0 \\
& \bar{M}_{e 2}-\bar{M}_{e 1}-\left(\bar{R}_{e 2}-\bar{R}_{e 1}\right)-\frac{1}{2} m r^{2} \omega^{2}\left(\eta_{1}^{2}-\eta_{2}^{2}\right) W_{s 0}=M r^{2} \omega^{2} W_{c} \sin \alpha
\end{aligned}
$$

Equation (3.8) 1 can be used to find the approximation of the rated speed $\omega$ when the motors operate in the synchronous state. The term of $\bar{M}_{e 1}+\bar{M}_{e 2}$ is the sum of the average electromagnetic torque of the two induction motors; the term of $\bar{R}_{e 1}+\bar{R}_{e 2}$ is the sum of the average friction torque of the two rotors; the other terms represent the load torque in the two rotors. Therefore, Eq. (3.8) $)_{1}$ is the equation of the vibrating torque between the rotors when the system 
operates in the steady state. Equation $(3.8)_{2}$ can be employed to search the approximations of the phase difference $\alpha$ when the motors are operating in the synchronous state. Therefore, Eq. $(3.8)_{2}$ is the relationship of the synchronous torque between the rotors and the difference of the residual torque between the two motors. According to Eq. $(3.8)_{2}$, the synchronous torque $T_{\text {Sys }}$ and the difference of the residual torque $T_{\text {Diff }}$ are specified as

$$
T_{\text {Sys }}=M r \omega^{2}\left|W_{c}\right| \quad T_{\text {Diff }}=T_{\text {Res } 2}-T_{\text {Res } 1}
$$

where

$$
T_{R e s 1}=\bar{M}_{e 1}-\bar{R}_{e 1}+\frac{1}{2} M r^{2} \omega^{2} \eta_{1}^{2} W_{s 0} \quad T_{R e s 2}=\bar{M}_{e 2}-\bar{R}_{e 2}+\frac{1}{2} M r^{2} \omega^{2} \eta_{2}^{2} W_{s 0}
$$

are considered as the residual torque in motor 1 and 2, respectively.

Substituting Eqs. (3.9) into Eq. (3.8) 1 , and rearranging it, the phase difference between the rotors can be expressed by

$$
\alpha=\arcsin \frac{T_{D i f f}}{T_{\text {Sys }} \operatorname{sgn}\left(W_{c}\right)}
$$

As shown in Eq. (3.10), the phase difference between the rotors is a function related to parameters $T_{\text {Diff }}$ and $T_{\text {Sys }}$. On account of $|\sin \bar{\alpha}| \leqslant 1$, the synchronous condition of the system is expressed by

$$
T_{\text {Sys }} \geqslant\left|T_{\text {Diff }}\right|
$$

According to Eq. (3.11), to guarantee synchronous operation between the rotors, the synchronous torques of the system should be greater than or identical with the difference of the residual torque between the two motors.

The coefficient of the synchronous ability of the system $\varsigma$ can be defined by

$$
\varsigma=\left|\frac{T_{L}}{T_{\text {Sys }}}\right|
$$

where $T_{L}$ is the maximum load torque in the two motors, i.e., $T_{L}=0.5 M r^{2} \omega^{2}\left[\left(\eta_{1}^{2}+\eta_{2}^{2}\right) W_{s 0}+\right.$ $2 W_{s}$ ]. The smaller the coefficient of the synchronous ability, the greater the possibility of implementing synchronous motion between the motors. When the value of $\varsigma$ is larger than 1 , it is difficult to implement synchronous operation between the rotors. The coefficient of the synchronous ability $\varsigma$ is related to the load torque $T_{L}$ and synchronous torque $T_{S y s}$, which is the function of parameters $\eta_{1}, \eta_{2}, r_{l}, \theta, \beta$ and $\zeta_{j}(j=x, y, z, \psi, \delta, v)$. As shown in Fig. 2, the coefficient of synchronous ability $\varsigma$ is decreased with an increase in the parameters $r_{l}$ and $\beta$, inversely, the coefficient $\varsigma$ is increased with an increase in the parameter $\theta$. In other words, the smaller the distance $l$ and angle $\beta$ between the two motors, the greater the possibility of implementing synchronous motion between the rotors; but the small value of angle $\theta$ is in favor of the implementation of synchronous operation in the vibrating system.

\subsection{Synchronous stability}

According to Eq. (3.10), two solutions of the phase difference $\alpha$ between the rotors can be obtained from numerical analysis, however, which one is a stable value should be discussed by the Hamilton principle. In light of Eqs. (2.5) and (2.6), Hamilton's action quantity during the period, represented by $S$, can be written as in the following

$$
S=\frac{1}{2 \pi} \int_{0}^{2 \pi} L d \varphi=\frac{1}{2 \pi} \int_{0}^{2 \pi}(T-V) d \varphi
$$


(a)

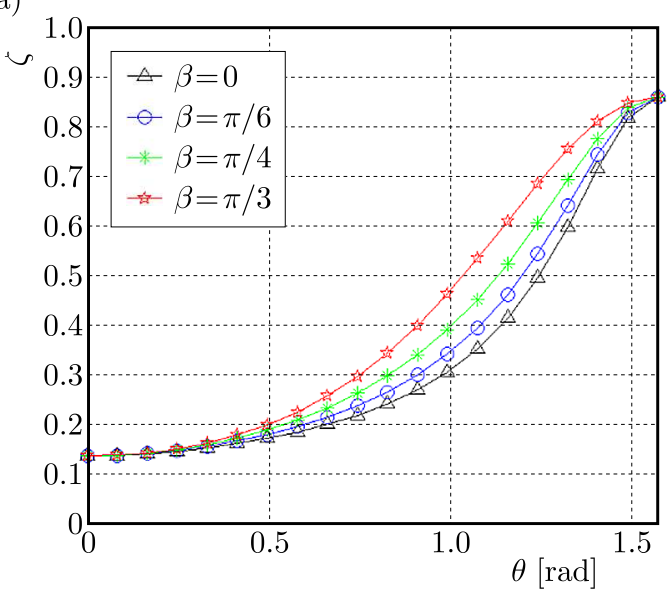

(c)

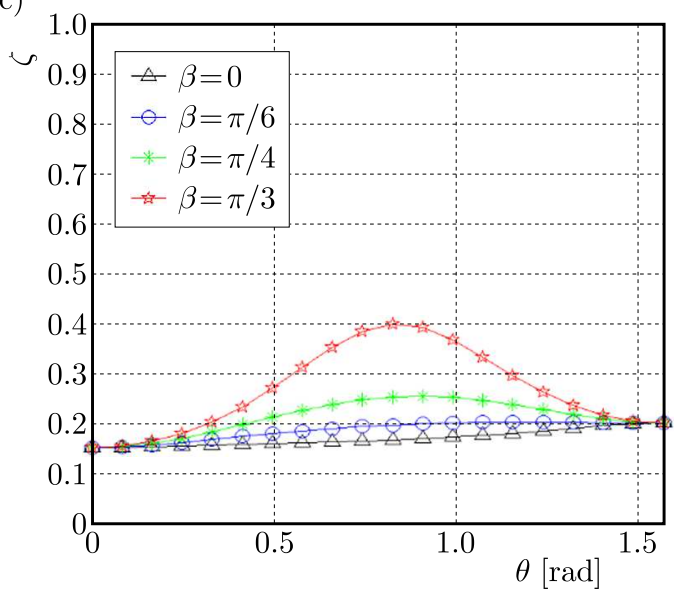

(b)

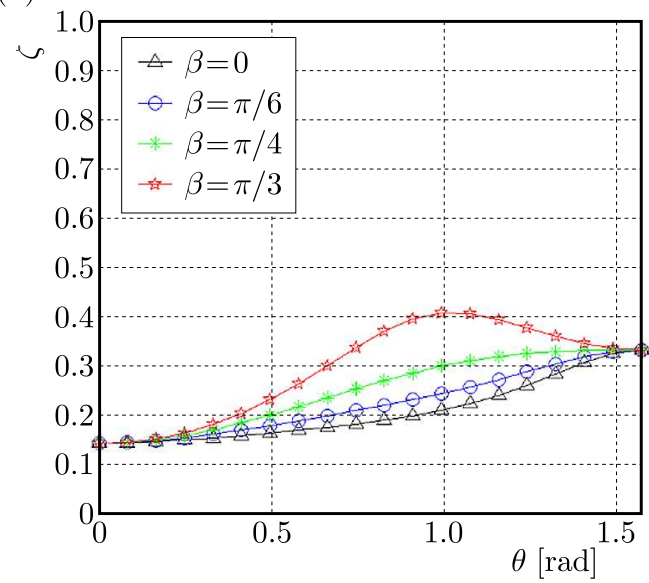

(d)

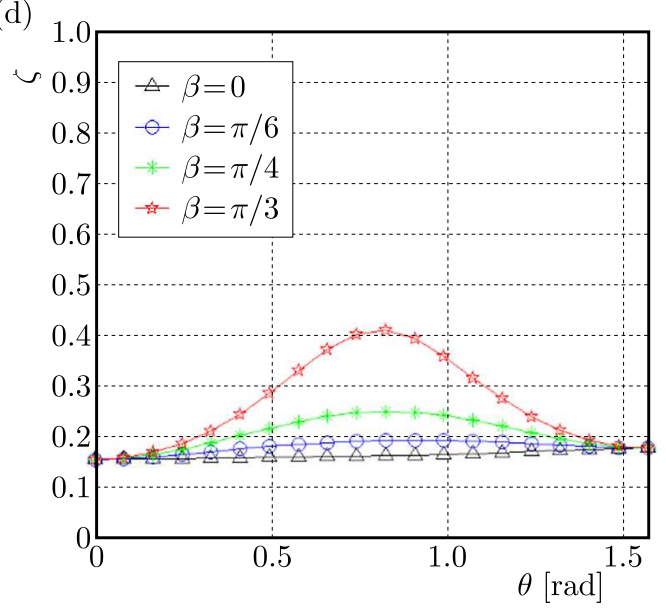

Fig. 2. Coefficients of the synchronous ability when $\eta_{1}=0.02$ and $\eta_{2}=0.04$; (a) $r_{l}=0.5$, (b) $r_{l}=1$, (c) $r_{l}=2$, (d) $r_{l}=3$

In light of Eq. (3.3), substituting the first-order derivative of Eq. (3.2) related to time $t$ into Eqs. (2.5) and (2.6), and Hamilton's action quantity $S$, one obtains

$$
S=-\frac{1}{4} m r^{2} \omega^{2} W_{c} \cos \bar{\alpha}
$$

According to (Kong et al., 2016a), the phase difference corresponding to Eq. (3.10) satisfied to the minimum Hamilton's action is stable. In other words, the second-order derivative of $S$ related to $\alpha$ in Eq. (3.13) should be greater than zero, i.e.

$$
\left.\frac{d^{2} S}{d \alpha^{2}}\right|_{\alpha=\alpha_{0}}>0
$$

Substituting Eq. (3.14) into Eq. (3.15), the condition of synchronous stability can be expressed as

$$
W_{c} \cos \alpha>0
$$

As shown in Eq. (3.15), if $W_{c}>0$, the phase difference $\alpha$ located in the region of $(-\pi / 2, \pi / 2)$ is stable; and if $W_{c}<0$, the phase difference $\alpha$ located in the region of $(\pi / 2,3 \pi / 2)$ is stable; when $W_{c}=0$, the parameters of the system cannot satisfy the synchronous condition in Eq. (3.11). 


\section{Numerical analysis}

In this Section, some numerical analysis is carried out to discuss the stable phase difference, which can be calculated according to Eqs. (3.10) and Eq. (3.16). The phase difference is determined by the parameters $T_{\text {Diff }}$ and $T_{\text {Sys }}$. These parameters are functions of frequency ratios $n_{j}$ $(j=x, y, z, \psi, \delta, v)$, damping ratios $\zeta_{j}$, mass ratios $\left(\eta_{1}, \eta_{2}\right)$ and geometrical parameters $(l, \beta, \theta)$. However, the synchronous state of the system is less influenced by the damping ratios as the damping coefficients are very small in the vibration screening machine. Moreover, the system is a far resonance system, and so the value of frequency ratios is greater than or equal to 5 . Therefore, mass ratios and geometrical parameters are important to the synchronous investigation in this paper. The values of the parameters corresponding to general engineering application are $n_{j}=5(j=x, y, z, \psi, \delta, v), \zeta_{j}=0.07, r_{l}=\{0.5,1,2,3\}$, and $\beta=\{0, \pi / 6, \pi / 4, \pi / 3\}$.

(a)

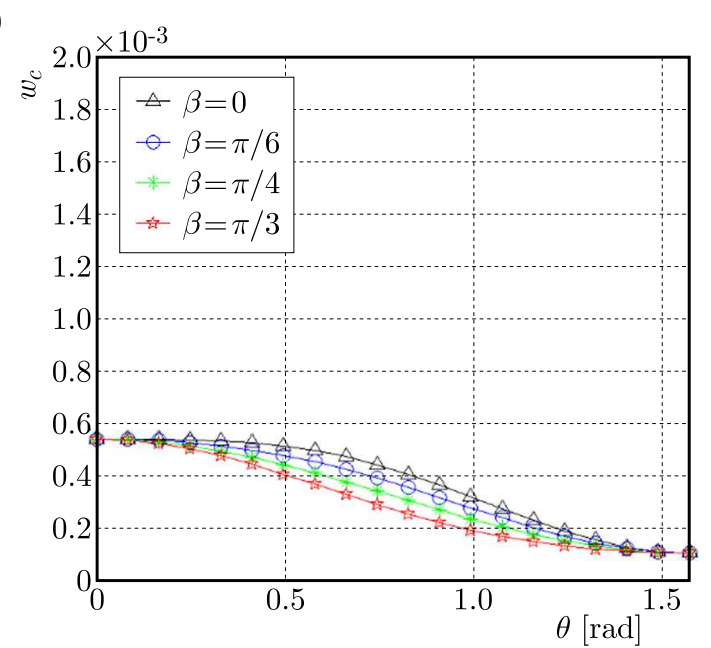

(c)

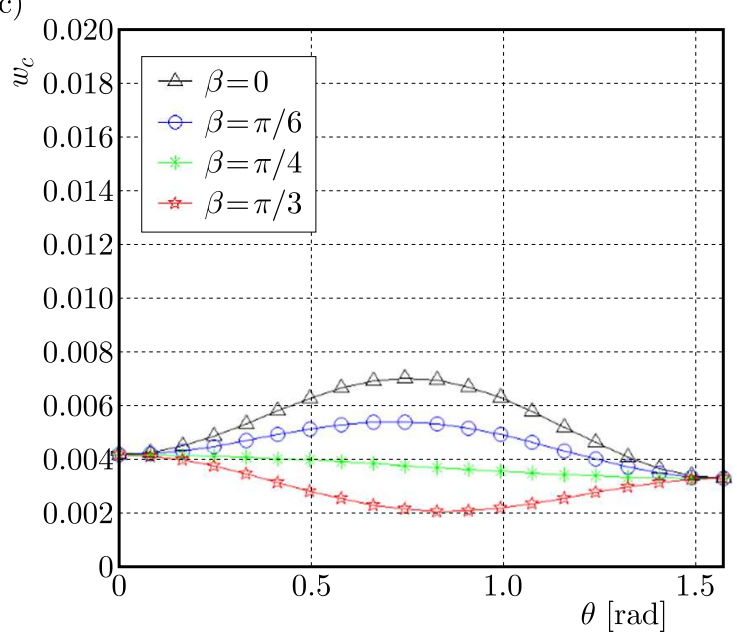

(b)

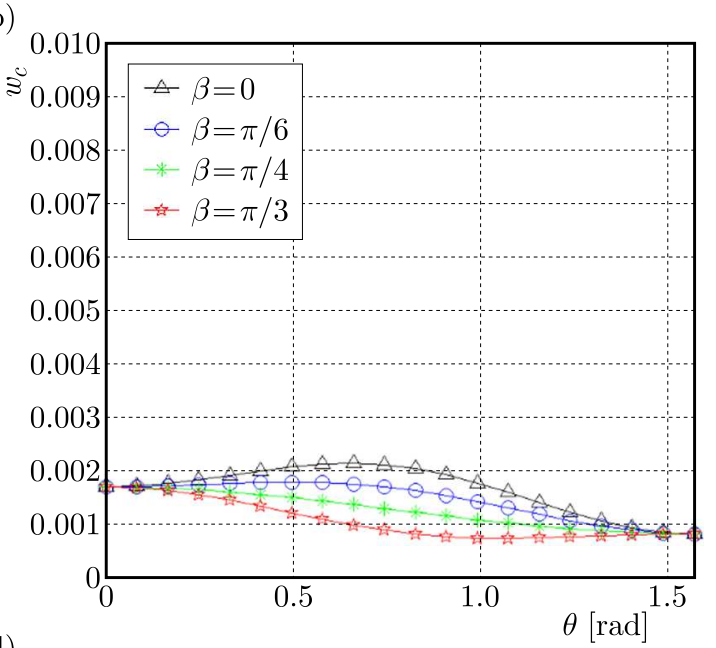

(d)

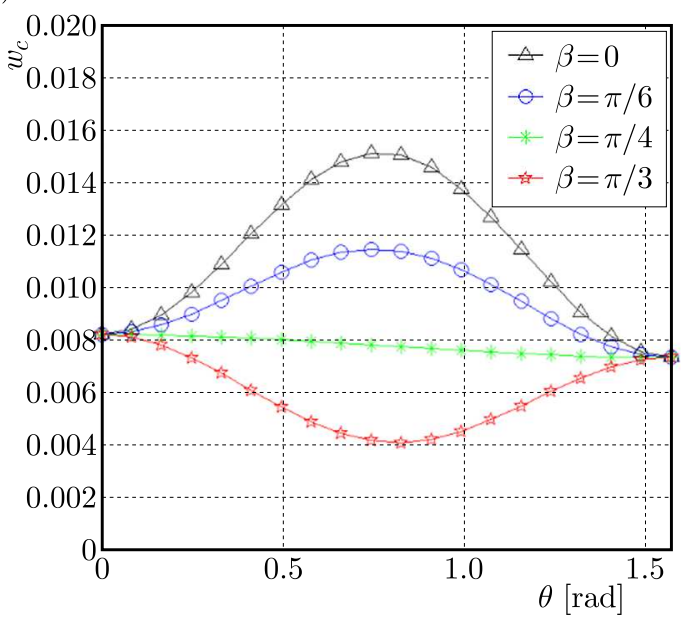

Fig. 3. Synchronous torque when $\eta_{1}=\eta_{2}=0.02$; (a) $r_{l}=0.5$, (b) $r_{l}=1$, (c) $r_{l}=2$, (d) $r_{l}=3$

Firstly, we considered mass of the unbalanced rotors to be identical, and so the mass ratios $\left(\eta_{1}, \eta_{2}\right)$ between the rotors and the vibrating body should be identical, i.e., $\eta_{1}=\eta_{2}$. Thus, Eq. (3.8) 2 is simplified as $\bar{M}_{e 2}-\bar{M}_{e 1}-\left(\bar{R}_{e 2}-\bar{R}_{e 1}\right)=M r^{2} \omega^{2} W_{c} \sin \alpha$. As the two motors are of the same type, the parameters of the motors are also alike. In this case, the difference of residual torque in motor 1 and 2 should be zero, i.e., $\bar{M}_{e 2}-\bar{M}_{e 1}-\left(\bar{R}_{e 2}-\bar{R}_{e 1}\right)=0$. Therefore, Eq. (3.8) 2 is further rewritten as $\sin \alpha=0$, and there are two solutions for $\alpha$ in this situation, i.e., $\alpha=0$ or $\alpha=\pi$, which is stable solution determined by Eq. (3.16). According to Eq. (3.16), the stability of the phase difference is influenced by the synchronous torque $W_{c}$. If $W_{c}<0$, the condition 
of synchronous stability of the system is replaced by $\cos \alpha<0$; if $W_{c}>0$, the stability of the system is replaced with $\cos \alpha>0$. Therefore, the synchronous torque $W_{c}$ is a key coefficient to determine the stability of the phase difference. The values of the synchronous torque between the two rotors are shown in Fig. 1 when $\eta_{1}=\eta_{2}=0.02$. As illustrated in this figure, the value of the synchronous torque $W_{c}$ is greater than zero when $\eta_{1}=\eta_{2}$. As a result, the condition of synchronous stability in the system can be expressed by $\cos \alpha>0$. On account of $\sin \alpha=0$, the phase difference between the motors is stabilized at $\alpha=0$. On the other hand, the synchronous torque $W_{c}$ is gradually increased with an increase in $r_{l}$, decreased with an increase in $\beta$, and first increased and then decreased with the increase of $\theta$.

Secondly, consider the mass of the unbalanced rotors to be different, and so the mass ratios $\left(\eta_{1}, \eta_{2}\right)$ of the rotors and the vibrating body should be nonequivalent, such as $\eta_{1}=0.02$ and $\eta_{2}=0.04$. Thus, Eq. (3.8) $)_{2}$ is further rewritten as $\sin \alpha=-\sin \left[\left(\eta_{1}^{2}-\eta_{2}^{2}\right) W_{s 0} / 2 W_{c}\right]$ on account of $\bar{M}_{e 2}-\bar{M}_{e 1}-\left(\bar{R}_{e 2}-\bar{R}_{e 1}\right)=0$, and there are two solutions for $\alpha$ in thid situation, i.e., $\alpha=-\arcsin \left[\left(\eta_{1}^{2}-\eta_{2}^{2}\right) W_{s 0} / 2 W_{c}\right]$ or $\left.\alpha=\pi+\arcsin \left(\eta_{1}^{2}-\eta_{2}^{2}\right) W_{s 0} / 2 W_{c}\right]$. The solution is stable and determined by Eq. (3.16). The stable phase difference between the motors in this case is illustrated in Fig. 4. It follows that the phase difference is influenced by parameters $r_{l}, \beta$ and $\theta$. The greater the parameters $\beta$ and $r_{l}$, the greater stable phase difference between the motors. However, the phase difference is irregularly fluctuated with changes in the parameter $\theta$. Of course, the stable phase difference can be also obtained when the mass ratio $\left(\eta_{1}, \eta_{2}\right)$ is equal to other values. As the space limitation, the peculiar example is only calculated numerically.

(a)

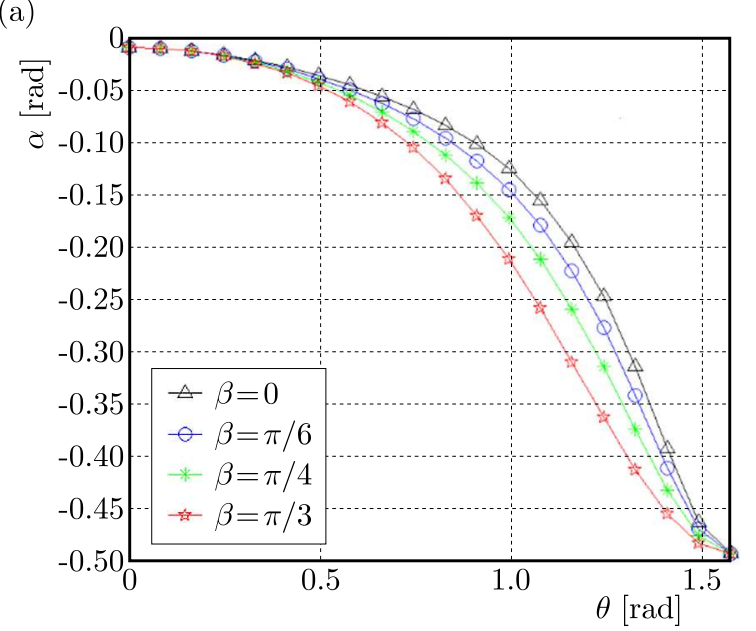

(c)

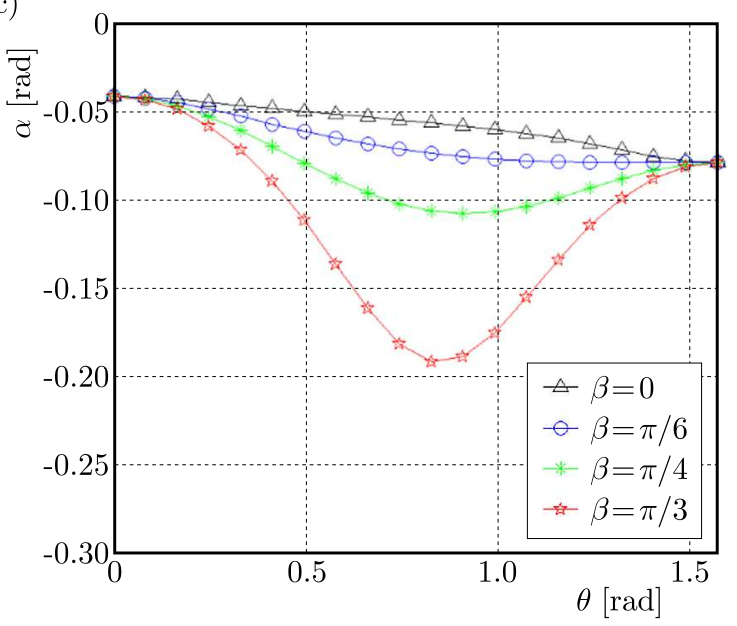

(b)

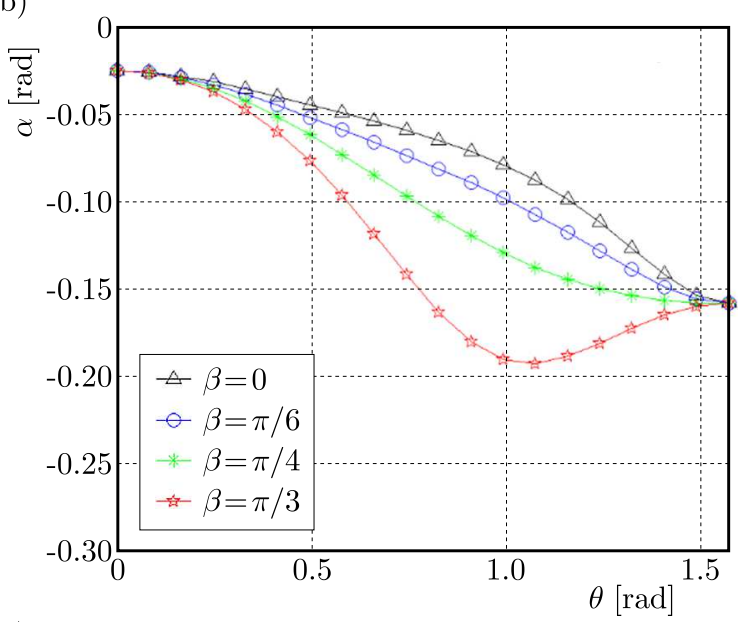

(d)

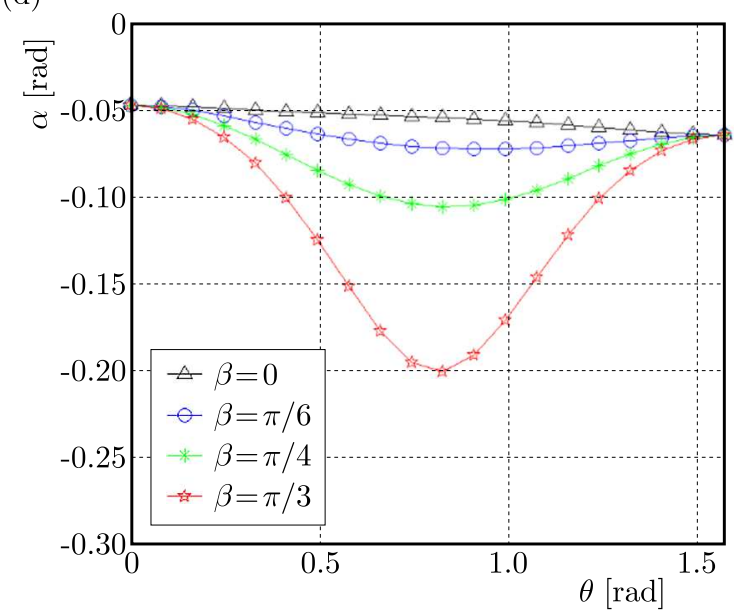

Fig. 4. Stable phase difference when $\eta_{1}=0.02$ and $\eta_{2}=0.04$; (a) $r_{l}=0.5$, (b) $r_{l}=1$, (c) $r_{l}=2$, (d) $r_{l}=3$ 
To understand variation tendencies of the key parameters of the system, the relationship among the phase difference, synchronization torque and angle $\theta$ should be discussed. Firstly, considering the mass of the unbalanced rotor to be identical, as shown in Figs. 5a and 5b, it can be seen that the phase difference is stabilized in zero forever, and the synchronization torque is gradually increased with an increase in the parameter $r_{l}$. Secondly, considering the mass of the unbalanced rotor to be different, it follows that the phase difference is no longer a single zero solution with the variation parameter $r_{l}$, but the synchronization torque is also gradually increased with an increase in the parameter $r_{l}$. The analysis further verifies that the synchronous state between the motors is mainly determined by the mass of the unbalanced rotors, and the synchronization torque is influenced by the distance between the motors and unrelated to the phase difference. Therefore, for designing the vibrating screen, such as the proposed model in this paper, the installation position between the motors and the mass of the rotors should be carefully chosen, in this case, the ideal dynamic characteristics of the system can be obtained.

(a)

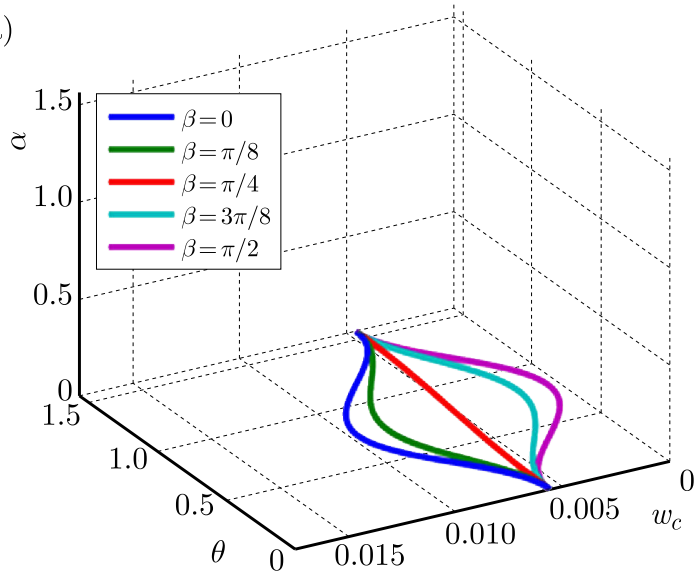

(c)

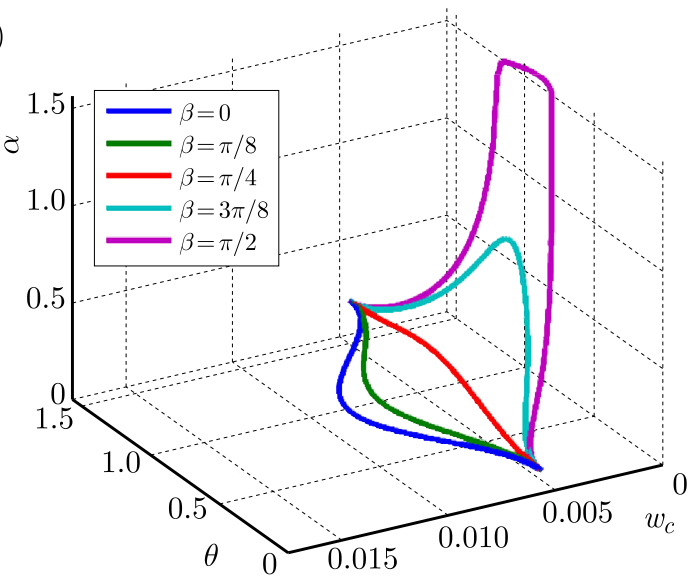

(b)

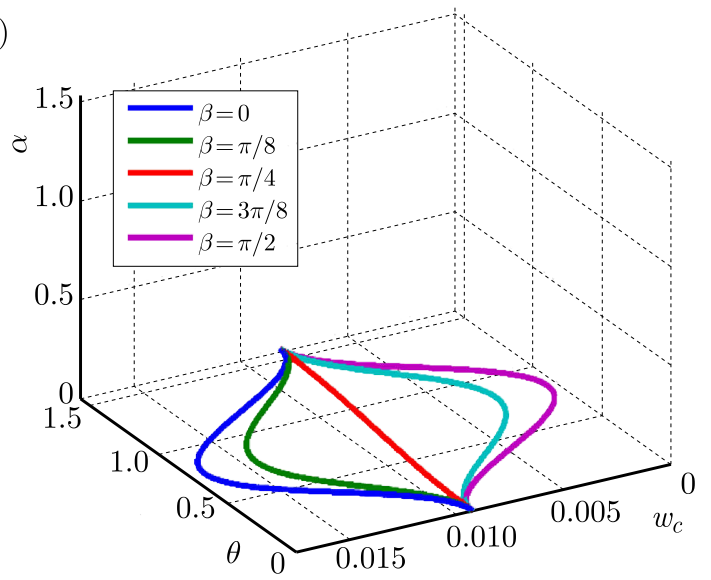

(d)

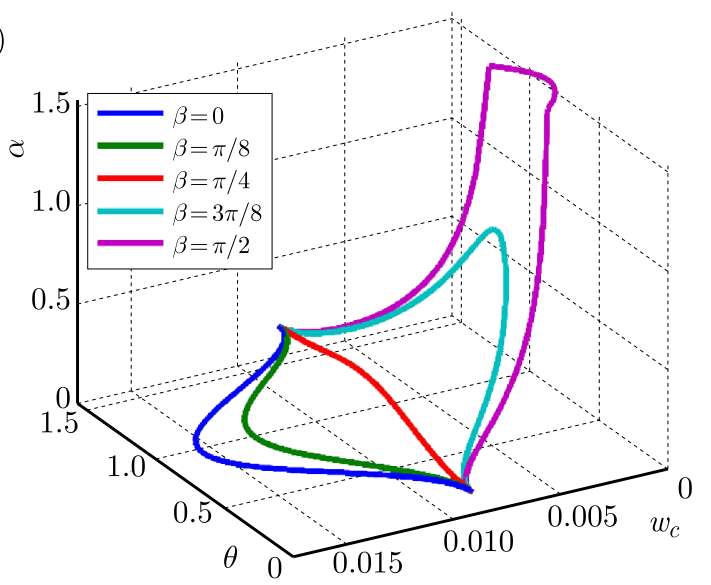

Fig. 5. The relationship among the phase difference, synchronization torque and angle $\theta$;

(a) $\eta_{1}=\eta_{2}=0.02, r_{l}=3$, (b) $\eta_{1}=\eta_{2}=0.02, r_{l}=4$, (c) $\eta_{1}=0.02, \eta_{2}=0.04, r_{l}=3$,

(d) $\eta_{1}=0.02, \eta_{2}=0.04, r_{l}=4$

According to Eqs. (3.3), (3.4) and (3.5), the dynamic characteristics of the vibrating body can be estimated with the stable phase difference between the rotors. Combining the theory of rigid body dynamics with the synchronous state between the rotors, the dynamic characteristics of the vibrating body can be also ascertained in light of Fig. 1. Table 1 shows oscillation of the vibrating body in different directions. Symbol N represents no vibration in the corresponding direction, oppositely, symbol Y represents vibration in the corresponding direction. It follows that when $\eta_{1}=\eta_{2}$, the vibrating body oscillates in the plane coordinate system $O y z$ and swung around the axis $x$. In this case, thw material on the vibrating body vibrates in the Oyz plane 
to the is benefit of material transport; when $\eta_{1} \neq \eta_{2}$, the vibrating body oscillates in the space coordinate system $O x y z$ and swung around axis $x, y$ and $z$. In this case, the material on the vibrating body moving in the space coordinate system $O x y z$ decreases the transport efficiency.

Table 1. Oscillation of the vibrating body

\begin{tabular}{|c|c|c|c|c|c|c|}
\hline \multirow{2}{*}{ Samples } & \multicolumn{6}{|c|}{ Direction } \\
\cline { 2 - 7 } & $x$ & $y$ & $z$ & $\psi$ & $\delta$ & $v$ \\
\hline \hline$\eta_{1}=\eta_{2}$ & $\mathrm{~N}$ & $\mathrm{Y}$ & $\mathrm{Y}$ & $\mathrm{Y}$ & $\mathrm{N}$ & $\mathrm{N}$ \\
\hline$\eta_{1} \neq \eta_{2}$ & $\mathrm{Y}$ & $\mathrm{Y}$ & $\mathrm{Y}$ & $\mathrm{Y}$ & $\mathrm{Y}$ & $\mathrm{Y}$ \\
\hline
\end{tabular}

\section{Computer simulations}

This Section refers to the case when the unbalanced rotors with the same mass are actuated by the motors in the far resonance system. The values of parameters are as follows: $k_{x} \approx k_{y} \approx k_{z}=$ $98596, k_{\psi} \approx k_{\delta} \approx k_{v}=19719, f_{x} \approx f_{y} \approx f_{z}=207, f_{\psi} \approx f_{\delta} \approx f_{v}=150, \theta=\pi / 4, \beta=\pi / 3$, $m_{1}=4, m_{2}=4, m_{3}=100, r=0.05, l=0.5, J_{\psi} \approx J_{\delta} \approx J_{v}=20$. The values of parameters are $\eta_{1}=0.04, \eta_{2}=0.04, \omega_{x} \approx \omega_{y} \approx \omega_{z} \approx \omega_{\psi} \approx \omega_{\delta} \approx \omega_{v}=31.4, \zeta_{x} \approx \zeta_{y} \approx \zeta_{z} \approx \zeta_{\psi} \approx \zeta_{\delta} \approx \zeta_{v}=0.07$, $r_{l}=1$. With implementation of computation simulations, the dynamic characteristics of the system are found and shown in Fig. 6. As can be seen in Fig. 6a, the velocities of the motors,

(a)

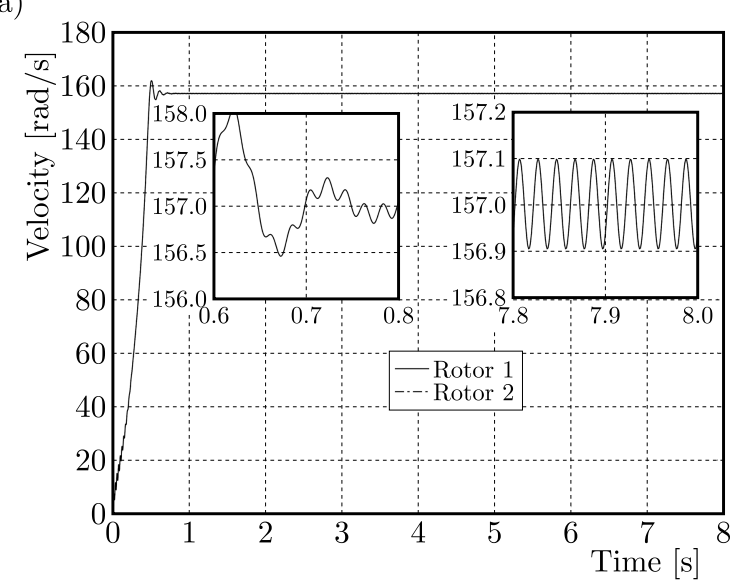

(c)
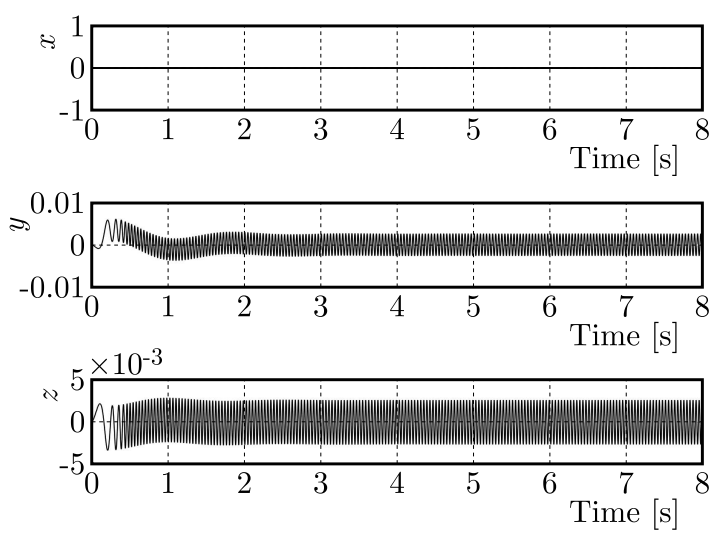

(b)

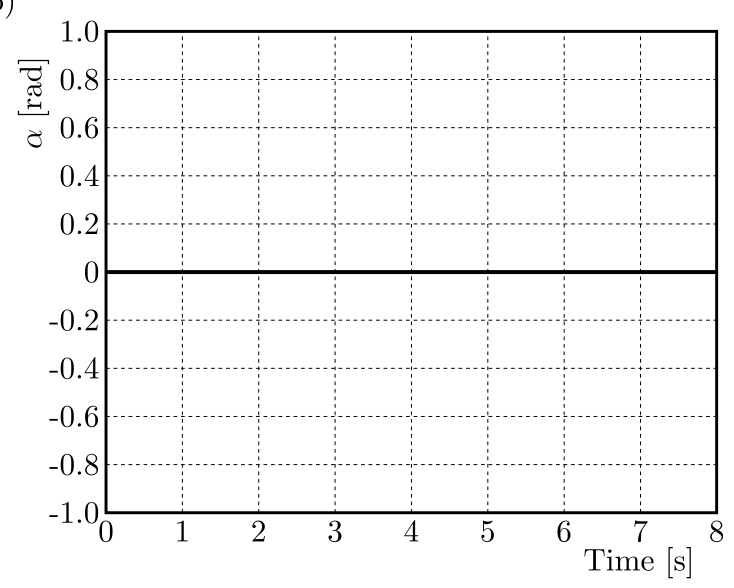

(d)
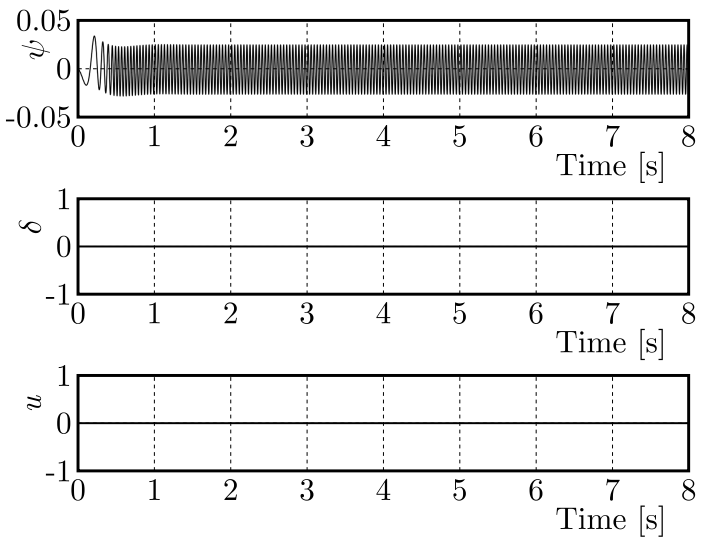

Fig. 6 . The dynamic characteristics of the system when $\eta_{1}=\eta_{2}=0.02$; (a) velocity of the motor,

(b) phase difference between the motors, (c) displacement of the vibrating body, (d) rotary oscillating of the vibrating body 
supplied by the electric source at the same time, are consistent in the total operation stage, and so the rotors actuated by the identical motors, are synchronous in such values. The phase difference between the rotors is stabilized at zero in the whole range, as shown in Fig. 6b, which is in good agreement with the theoretical results from Section 4. In this case, the displacement of the vibrating body in the $x-, y$-, and $z$-directions is shown in Fig. 6c. As the stable phase difference is always located in zero, the amplitude of the vibrating body in the $x$-direction is zero, therefore, the vibrating body driven with the motors oscillates in the plane Oyz. The driving force, produced by the unbalanced rotors, may result in rotary oscillation of the vibrating body, such as shown in Fig. 6 d. The rotary amplitudes of the vibrating body in the $\delta$ - and $v$-directions are zero, however, the body oscillates in the $\psi$-direction (around axis $x$ ) due to the zero phase between the rotors. It can be seen from the analysis above, the vibration screening machinery oscillates in the $O y z$ plane and swung around axis the $x$ when the stable phase difference between the rotor is stabilized in zero.

(a)

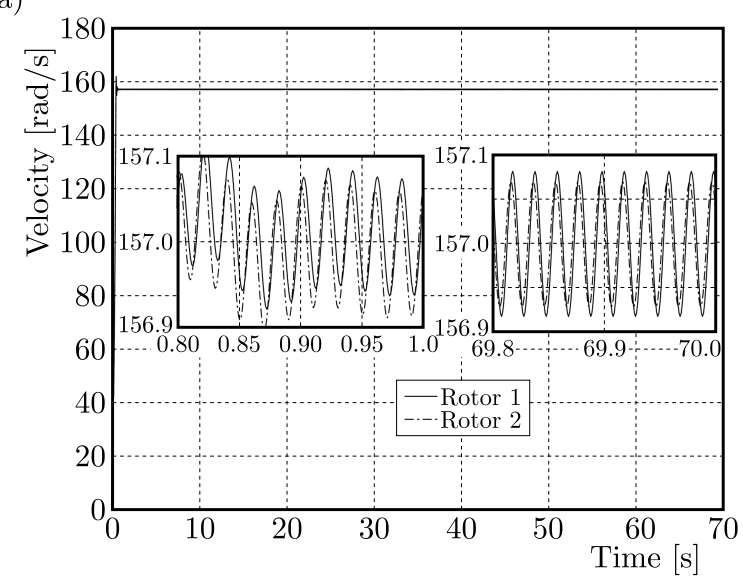

(c)
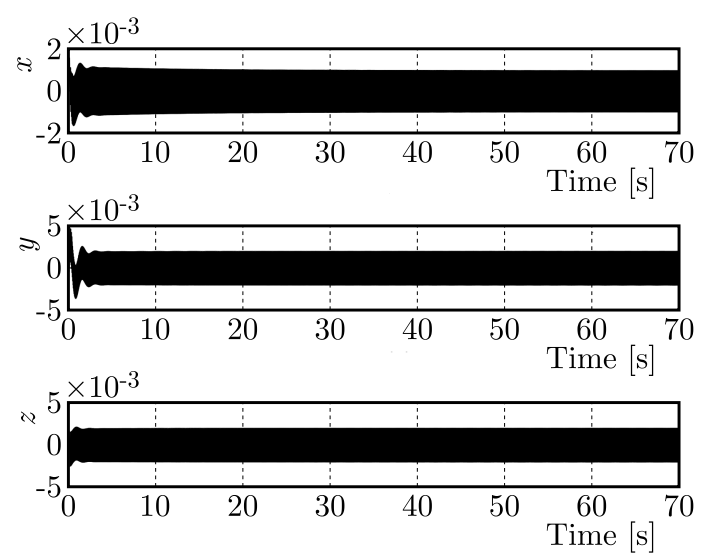

(b)

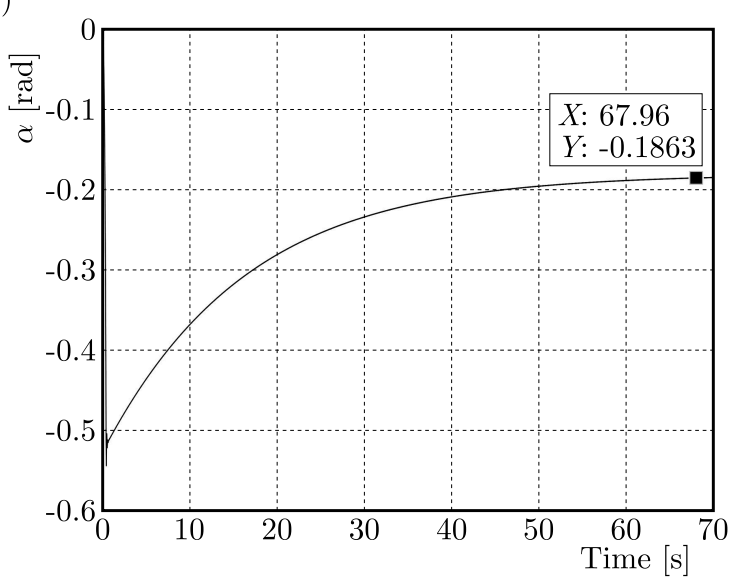

(d)
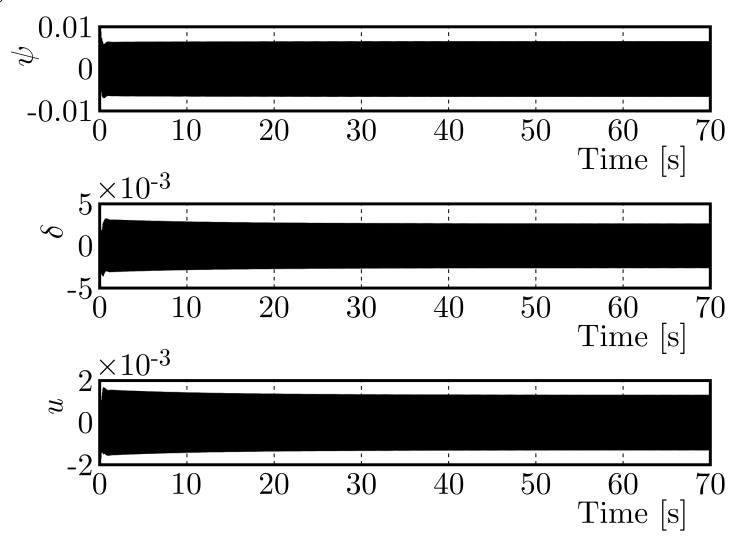

Fig. 7. The dynamic characteristics of the system when $\eta_{1}=0.02$ and $\eta_{2}=0.04$; (a) velocity of the motor, (b) phase difference between the motors, (c) displacement of the vibrating body, (d) rotary oscillating of the vibrating body

To verify correctness of the theoretical analysis, some numerical simulations are carried out. This Section refers to the case when the unbalanced rotors with different mass are actuated by the two motors in the far resonance system. The values of parameters are following: $k_{x} \approx k_{y} \approx$ $k_{z}=98596, k_{\psi} \approx k_{\delta} \approx k_{v}=19719, f_{x} \approx f_{y} \approx f_{z}=207, f_{\psi} \approx f_{\delta} \approx f_{v}=150, \theta=\pi / 4, \beta=\pi / 3$, $m_{1}=4, m_{2}=2, m_{3}=100, r=0.05, l=0.5, J_{\psi} \approx J_{\delta} \approx J_{v}=20$. The values of parameters corresponding to Eq. (3.1) are $\eta_{1}=0.04, \eta_{2}=0.02, \omega_{x} \approx \omega_{y} \approx \omega_{z} \approx \omega_{\psi} \approx \omega_{\delta} \approx \omega_{v}=31.4$, $\zeta_{x} \approx \zeta_{y} \approx \zeta_{z} \approx \zeta_{\psi} \approx \zeta_{\delta} \approx \zeta_{v}=0.07, r_{l}=1$. The dynamic characteristics of the system are shown 
in Fig. 7. As can be seen in Fig. 7a, the velocities of the motors, supplied by the electric source at the same time, are different at the start stage as the rotational inertia of the rotor is strikingly different. It should be noted that the velocities of the two rotors are compatible to each other in at steady operation stage. The phase difference between the rotors is stabilized at -0.1863 at the synchronous stage, as shown in Fig. 7b, which is in good agreement with the theoretical analysis (Fig. 4b). The displacement of the vibrating body in the $x-, y$-, and $z$-directions is shown in Fig. 7c. The stable phase difference is nonzero, the vibrating body is vibrating in the $x^{-}, y$-, and $z$-directions; therefore, the vibrating body driven with the motors oscillates in the space coordinate system Oxyz. The driving forces, produced by the unbalanced rotors, result in rotary oscillation of the vibrating body, as shown in Fig. 7d. The vibrating body oscillates in the $\psi$-, $\delta$ - and $v$-directions on account of the nonzero phase between the rotors. It can be seen from analysis above that the vibration screening machine oscillates in the $O x y z$ plane and swung around the axis $x, y$ and $z$ when the stable phase difference between the rotor is stabilized in nonzero.

\section{Conclusions}

In this paper, two unbalanced rotors excited with paralleled and counterrotating motors in a far resonance system are concerned, whose dynamic characteristics are considered as spatial synchronization on account of the existence of spatial installation of the motors. Based on the dynamic equation of the system, the synchronous condition between the rotors is derived with the Poincaré method. According to the synchronous condition, to guarantee synchronous operation of the rotors, the synchronous torques between the motors should be greater than or identical with the difference between the residual torques of the two motors. Two solutions of the phase difference can be obtained through solving the synchronous condition, however, whether it is stable can be deduced by the Hamilton principle. Therefore, the synchronous stability is determined by the synchronous torque, i.e., if the synchronous torque is greater than zero, the phase difference located in the region $(-\pi / 2, \pi / 2)$ is stable. If the synchronous torque is smaller than zero, the phase difference $\alpha$ located in the region $(\pi / 2,3 \pi / 2)$. When the synchronous torque is equal to zero, the parameters of the system cannot satisfy the synchronous condition. According to the stable phase difference between the rotors, the dynamic characteristics of the vibration screening machine can be predicted. The research result shows that the vibration screening machine actuated with the unbalanced rotors of identical mass exibits planar motion as the stable phase difference between the rotors is stabilized in zero. The vibration screening machine actuated with the unbalanced rotors of various mass moves spatially as the stable phase difference is stabilized in a nonzero value.

\section{Acknowledgements}

National Natural Science Foundation of China (Grant No. 51705437), Strategic Cooperation Project of Science and Technology between SWPU and Nanchong (No. 18SXHZ0016), Scientific Research Starting Project of SWPU (No. 2017QHZ009).

\section{References}

1. Balthazar J., Felix J., Brasil R., 2004, Short comments on self-synchronization of two non-ideal sources supported by a flexible portal frame structure, Journal of Vibration and Control, $J V C, \mathbf{1 0}, 12,1739-1748$

2. Balthazar J., Felix J., Brasil R., 2005, Some comments on the numerical simulation of self-synchronization of four non-ideal exciters, Applied Mathematics and Computation, 164, 2, 615-625 
3. Banaszewski T., Schollbach A., 1998, Vibration analysis of machines with self-synchronizing unbalance-type exciters, Aufbereitungs-Technik, 39, 8, 383-393

4. Blekhman I.I., 1988, Synchronization in Science and Technology, ASME Press, New York

5. Chen X.Z., Kong X.X., Zhang X.L., Li L.X., Wen B.C., 2016, On the synchronization of two eccentric rotors with common rotational axis theory and experiment, Shock and Vibration, 2016

6. Czolczynski K., Perlikowski P., Stefanski A., Kapitaniak T., 2012, Synchronization of pendula rotating in different directions, Communications in Nonlinear Science and Numerical Simulation, 17, 9, 3658-3672

7. Czolczynski K., Perlikowski P., Stefanski A., Kapitaniak T., 2013, Synchronization of the self-excited pendula suspended on the vertically displacing beam, Communications in Nonlinear Science and Numerical Simulation, 18, 2, 386-400

8. FAng P., Hou Y.J., 2018, Synchronization characteristics of a rotor-pendula system in multiple coupling resonant systems, Proceedings of The Institution of Mechanical Engineers Part C - Journal of Mechanical Engineering Science, 232, 10, 1802-1822

9. FAng P., Yang Q.M., Hou Y.J., Chen Y., 2014, Theoretical study on self-synchronization of two homodromy rotors coupled with a pendulum rod in a far-resonant vibrating system, Journal of Vibroengineering, 16, 5, 2188-2204

10. Hou Y.J., Du M.J., Fang P., Zhang L., 2018, Synchronization and stability of an elastically coupled tri-rotor vibration system, Journal of Theoretical and Applied Mechanics, 55, 1, 227

11. Inoue J., Araki Y., MiYaura S., 1951, Self-synchronization of mechanical system (multiple cycle), Proceedings of Japanese Mechanical Engineering Society, 42, 103-110

12. Kapitaniak M., Czolczynski K., Perlikowski P., Stefanski A., Kapitaniak T., 2014, Synchronous states of slowly rotating pendula, Physics Reports, 541, 1, 1-44

13. Kong X.X., Zhang X.L., Chen X.Z., Wen B.C., Wang B., 2016a, Phase and speed synchronization control of four eccentric rotors driven by induction motors in a linear vibratory feeder with unknown time-varying load torques using adaptive sliding mode control algorithm, Journal of Sound and Vibration, 370, 23-42

14. Kong X.X., Zhang X.L., Chen X.Z., Wen B.C., Wang B., 2016b, Synchronization analysis and control of three eccentric rotors in a vibrating system using adaptive sliding mode control algorithm, Mechanical Systems and Signal Processing, 72-73, 432-450

15. Nanha Djanan A.A., Nana Nbendjo B.R., Woafo P., 2013, Electromechanical control of vibration on a plate submitted to a non-ideal excitation, Mechanics Research Communications, $\mathbf{5 4}, 72-82$

16. NAnha Djanan A.A., NAna Nbendjo B.R., WoAfo P., 2014, Effect of self-synchronization of DC motors on the amplitude of vibration of a rectangular plate, The European Physical Journal Special Topics, 223, 813-825

17. Paz M., Cole J., 1992, Self-synchronization of two unbalanced rotors, Journal of Vibration and Acoustics, 114, 1, 37-41

18. Sperling L., Ryzhik B., Linz C., Duckstein H., 2000, Simulation of two-plane automatic balancing of a rigid rotor, 2nd International Conference on Control of Oscillations and Chaos (COC-2000), St. Petersburg, Russia: Elsevier

19. TAng L.P., Zhu X.H., Li J.H., 2019, Effects of the synchronous variation of the static and the kinetic friction coefficients on stick-slip vibration of drillstring, Iranian Journal of Science and Technology, Transactions of Mechanical Engineering, 43, 275-283, DOI: 10.1007/s40997-018-0156-y

20. Wen B.C., FAn J., Zhao C.Y., 2009, Synchronization and Controled Synchronization in Engineering, Science Press, Beijing

21. Xino J.Y., Zhong S.M., Li Y.T., Xu. F., 2017, Finite-time Mittag-Leffler synchronization of fractional-order memristive BAM neural networks with time delays, Neurocomputing, 219, 431-439 
22. Zhang X.L., Wen B.C., Zhao C.Y., 2014, Vibratory synchronization transmission of two exciters in a super-resonant vibrating system, Journal of Mechanical Science and Technology, 28, 6, 2049-2058

23. Zhang X.L., Wen B.C., Zhao C.Y., 2016, Theoretical study on synchronization of two exciters in a nonlinear vibrating system with multiple resonant types, Nonlinear Dynamics, 85, 1, 141-154

24. Zhang X.L., Wen B.C., Zhao C.Y., 2017, Vibratory synchronization transmission of a cylindrical roller in a vibrating mechanical system excited by two exciters, Mechanical Systems and Signal Processing, 96, 88-103

25. Zhao C.Y., Zhang Y.M., Zhang X.L., 2010a, Synchronisation and general dynamic symmetry of a vibrating system with two exciters rotating in opposite directions, Chinese Physics B, 19, 3

26. Zhao C.Y., Zhu H.T., Zhang Y.M., Wen B.C., 2010, Synchronization of two coupled exciters in a vibrating system of spatial motion, Acta Mechanica Sinica, 26, 477-493

Manuscript received July 23, 2018; accepted for print April 3, 2019 